\title{
Sistematización de experiencia Aprendizajes con anclaje En la convivencia ciudadana
}

\section{Systematization of experience Learning with anchorage In citizen coexistence}

\author{
Juan Diego Castrillón Cordovez \\ Universidad Autónoma de Occidente \\ https://orcid.org/0000-0002-2499-6215
}

\begin{tabular}{|l|l|l|}
\hline Recepción/Submission: & $\begin{array}{c}\text { Evaluación de contenidos/ } \\
\text { Peer-review outcome: }\end{array}$ & Aprobación/Acceptance: \\
\hline Enero (January) de 2019 & Febrero (February) de 2019 & Abril (April) de 2019 \\
\hline
\end{tabular}

\section{Cómo citar.}

Castrillón Cordovez, J. D. (2020). Sistematización de experiencia Aprendizajes con anclaje en la convivencia ciudadana. Revista Científica Sabia, 6(1). pp.112-126. Doi: https://doi.org/10.47366/ sabia.v6n1a7

Resumen. Este escrito ofrece una sistematización de experiencias docentes que se ordenó a identificar estrategias de aprendizaje para promover la convivencia ciudadana en contexto de conflictos.

Las experiencias tienen en cuenta enfoques determinados tanto por principios como resultados esperados desde consideraciones al estudiantado como centro del proceso de aprendizaje. Se ofrece como referente inmediato la reseña de actividades realizadas en tiempo de pandemia del Coronavirus con la invitación a la escucha activa grupos musicales y de cantantes con mensajes para favorecer la convivencia, como contextos para aplicar perspectivas de Pierre Bourdieu y Gianni Vattimo en particular orientados en clave global a quitarle el velo a prejuicios y estereotipos considerando que éste fue un objetivo modular.

Palabras clave. Convivencia Ciudadana; Competencia De La Escucha; Aprendizajes Desde Contextos.

\begin{abstract}
This written offers a systematization of teaching experiences that was ordered to identify learning strategies to promote citizen coexistence in the context of conflicts. The experiences take into account both principle-determined approaches and expected outcomes from considerations to the student body as the centre of the learning process. The review of activities carried out in the time of the Coronavirus pandemic is offered as an immediate reference, with the invitation to active listening of musical groups and singers with messages to favour coexistence, as contexts to apply the perspectives of Pierre Bourdieu and Gianni Vattimo in particular, oriented in a global key to remove the veil of prejudices and stereotypes, considering that this was a modular objective.
\end{abstract}

Keywords. Citizen Coexistence; Listening Competition; Learning From Contexts. 


\section{Introducción}

El reto que aquí se asumió en esta sistematización fue caracterizar un modo de intervención pedagógica en perspectiva de deberes y derechos para la convivencia ciudadana, dimensionada en Colombia como una competencia en el sentido de capacidad humana en respuesta a las circunstancias históricas.

Este reto implicó admitir los límites de una práctica de aprendizaje usualmente de orden intelectivo memorístico, y considerando además la necesidad de responder a una demanda social a las instituciones educativas, que pueda valerse además de las ciencias del comportamiento y de la evaluación rigurosa de modo que pueda ofrecer el apoyo requerido por los estudiantes de educación secundaria y superior en su búsqueda de habilidades de comprensión y de desempeño en el ejercicio ciudadano.

Esta orientación integral está alineada con un principio orgánico que incluye los aspectos cognitivo, psicomotor y afectivo, los cuales se requieren en diversos entornos educativos en cualquier edad. Con ella se pretende advertir los riesgos del enfoque educativo cuando le da un peso exclusivo a la racionalidad la cual es necesario reconocer como muy limitada en situaciones reales donde inciden aspectos prácticos, actitudinales, donde la racionalidad no puede ejercerse en forma absoluta y en todo momento. Adicionalmente este enfoque a los aprendizajes convoca a la sociedad civil como agente moral en una apertura a la sensibilidad y a la estética y a los afectos. En tal sentido este enfoque es coherente con el ciclo de aprendizaje que implica procesos inseparables de, respuesta y actuación (UNESCO, 2015) ${ }^{1}$

Desde esta perspectiva el método que se siguió fue el fenomenológico, pues consideró los eventos de aprendizaje tomando distancia de hacer caracterizaciones de una supuesta objetividad evaluable y una subjetividad descartable. Adicionalmente se tuvo en cuenta la convivencia ciudadana nó como una línea de desempeño planteado por el Estado, el gobierno o los partidos u otras instituciones sino como la situación vital compartida ordenada al desarrollo personal y social en articulación con modelos éticos y políticos pluralistas.

Al referirse a la convivencia entendida como la vida en relación con otros, (del latín, cum- vivere) compromete la responsabilidad compartida tanto personal como institucional para el buen vivir en un tejido social.

Invita a vivir juntos, a conocernos a nosotros mismos a través de la relación con otros, trabajando en proyectos motivadores en el respeto a los derechos humanos.

Su aprendizaje durante la formación en ciencias sociales y humanas compromete el reconocimiento de diversos contextos e historias de cada una de las personas, grupos e instituciones sociales, que en el caso de una de los enfoques aplicados se propuso reflexionar sobre la superación de los prejuicios étnicos, de orientación política, religiosa o de género, entre otros.

A modo de contraste con la tradición pedagógica centrada en la educación y medición de capacidades intelectuales es posible diferenciar, como aquí se hace, aprendizajes por sus dimensiones conceptuales, prácticas y actitudinales que apuntan a la comprensión del saber hacer en distintos contextos, a la vida compartida alrededor de intencionalidades, voluntades, actitudes, afectos que son determinantes en asuntos locales, regionales y globales, que convocan al pensamiento crítico y a la valoración de la interdependencia e interconexión entre diferentes países y poblaciones. Conlleva a un sentido de pertenencia a una humanidad común, más pacífica y sostenible, compartiendo valores y

\footnotetext{
${ }^{1}$ UNICEF Canada, 2011, Educating For Global Citizenship: A Practical Guide for Schools in Atlantic Canada.
} 


\section{Sistematización de experiencia Aprendizajes con anclaje En la convivencia ciudadana Juan Diego Castrillón Cordovez}

responsabilidades, empatía, solidaridad y respeto por las diferencias y la diversidad (UNESCO, 2015) ${ }^{2}$. En el ámbito pedagógico se alude a los aprendizajes de la convivencia ciudadana considerando la urgencia al aprender a saber, hacer, ser y vivir juntos (Delors, 1994) ${ }^{3}$.

En primer lugar se alienta la realización de prácticas de aprendizaje centrado en el estudiante y la consideración del enfoque en el desarrollo de capacidades prácticas y actitudinales además de las intelectuales. Estas capacidades se requieren considerando circunstancias locales y globales en el cual puede tomarse como referencia el diagnóstico consensuado por la Organización de Naciones Unidas, ONU y que se sistematizó en el planteamiento de los Objetivos de Desarrollo Sostenible para el período 2015-2030. Dado que es posible alinear situaciones por resolver con alguno de los 17 Objetivos de Desarrollo Sostenible, ODS, los docentes pueden adecuar e implementar estrategias y proyectos de aprendizaje teórico, práctico y actitudinal con sus propios estudiantes. Como cualquier investigación, las variaciones de los enfoques pueden aumentar o disminuir el efecto de la intervención. Los lectores deben tener esto en cuenta si buscan adaptar las intervenciones a su contexto particular.

1.1 Las prácticas han de tomar en cuenta los dominios o niveles de aprendizaje reconocidos entre otros por la Unesco, a los cuales corresponden evidencias, atributos claves y tópicos. Con base en estos tópicos y de acuerdo con la edad y el nivel de aprendizaje requerido se desarrollan los objetivos específicos. Si la comprensión de la convivencia ciudadana como propósito de aprendizaje involucra la promoción de procesos cognitivos, prácticos y actitudinales socioemocionales-, que implican además los planos estéticos y afectivos, además de los intelectuales, sus dominios son explicitados asít:

- Cognitivo: En él se mide el nivel de conocimiento de análisis, síntesis y comprensión desde un pensamiento informado y de lectura crítica necesario referido a los tópicos del ámbito local, regional y global, sistemas y estructuras y los asuntos que afectan la interacción y conexiones en distintos niveles comunitarios.

- Práctico. Se evalúa el sentido de pertenencia, de valores e identidad compartidos con base en los derechos humanos, referidos a tópicos tales como la construcción de identidad, relaciones y sentido de pertenencia de la gente a distintas comunidades y cómo éstas pueden conectarse en respeto de la diferencia y la diversidad.

- Socioemocional: Se evalúa la disposición del estudiante a nivel local, regional y global referida A tópicos o acciones que pueden ser tomadas individual o colectivamente que permiten que los alumnos se desarrollen afectiva, psicosocial y física y para permitirles vivir juntos con otros Respetuosa y pacíficamente.

1.2. Como referencia adicional se tuvieron en cuenta algunos hallazgos del Departamento de Negocios, Innovación y Habilidades del gobierno británico y de un equipo de disciplinas de comportamiento aplicado a evaluar el aprendizaje que identificó cuatro principios que los profesores y los encargados de formular políticas educativas en las instituciones pueden implementar para mejorar la asistencia a clases (tasas de retención) y el logro de objetivos de aprendizaje.

2 UNESCO, 2015, Global Citizenship Education: Topics And Learning Objectives, Open Access Repository (http://www.unesco.org/open-access/terms-use-ccbysa-en, 7, place de Fontenoy, 75352 Paris 07 SP, France

${ }^{3}$ Delors, Jacques (1994). "Los cuatro pilares de la educación", en La Educación encierra un tesoro. México: El Correo de la UNESCO, pp. 91-103.

${ }^{4}$ UNESCO, 2015, obra citada. Página 29 a 36. En este documento de la UNESCO se diferencian objetivos más específicos para primaria, secundaria y educación superior por cada tópico enunciado correspondiente al dominio cognitivo, práctico y actitudinal. 
El equipo gubernamental para diseñar propuestas de desempeño pedagógico para mejorar las tasas de retención y éxito motivó la creación del Centro de Investigación de Conductual para el desarrollo de Habilidades y Conocimiento de Adultos, The Behavioural Research Centre for Adult Skills and Knowledge (ASK, por su sigla en inglés) ${ }^{5}$. Finalmente se orientaron a valorar diferentes modos de apoyar el aprendizaje en la enseñanza de habilidades en inglés y matemáticas en Inglaterra ${ }^{6}$.

1.3 Se tiene en cuenta el alineamiento de los objetivos de los aprendizajes que apuntan al desarrollo de capacidades y competencias, con los procesos evaluativos orientados a evidenciar el alcance de distintos niveles de complejidad. Si se sigue el modelo pedagógico a partir de una Estructura de Resultados Observados de Aprendizaje (Bigs, 2006) ${ }^{7}$ donde se distinguen 4 niveles: el uniestructural, que identifica fechas, números, datos específicos. El multiestructural que permite realizar inferencias dentro de un mismo marco de observación. El nivel relacional que indaga por la elaboración de hipótesis explicativas, causas. nexos entre eventos que corresponden a distintos marcos de observación, y el nivel abstracto ampliado, que permite emitir juicios desde una posición sustentada, devela posiciones ideológicas y remite a diversos correlatos socioculturales o disciplinares y los pone en diálogo.

\section{El riesgo del enfoque racionalista desde principios}

Una retrospectiva desde las ciencias sociales muestra que hoy es necesario superar el excesivo optimismo por la razón dando espacio a otras dimensiones de la condición humana, requiriéndose un giro incluyente donde al lado de las aptitudes racionales teóricas se ubique la practicidad, los afectos y valores, la cual requiere considerar el aula como un espacio abierto y seguro donde los participantes puedan asumir diversas posiciones reflexivas y críticas que respeten el diálogo respetuoso y promuevan una transformación social positiva.

El enfoque a partir de principios tiene el riesgo de quedarse en un sesgo racionalista, idealista, que no pondere resultados sino la racionalidad y las intenciones, como fue desarrollado en la modernidad por la filosofía alemana, en particular por Frederick Hegel y Emmanuel Kant. Está vigente cuando se el reconocimiento del interés universal racional expresado en ideas y porque señala como puntos de partida o imperativos los siguientes: 1.- Debo obrarse como todo mundo debiera obrar si estuviera en mi circunstancia. 2.- Las personas son fines, de ninguna manera son usables como medios. La vigencia de este enfoque es cuestionable en cuanto que señala un marco racional universal como fuente de actuación lo cual contradice la existencia de varios modelos racionales con múltiples perspectivas de interpretación, que además no es la razón la única fuente de moralidad sino los afectos y finalmente que se requieren también resultados que confirmen la conveniencia de y validez los principios. Este aspecto ético es una clave para considerar pues referirse a la convivencia ciudadana corresponde a los ciudadanos como agentes morales motivados tanto por el deber ser desde los principios y considerando además medios y resultados.

Adicionalmente pueden plantearse intervenciones pedagógicas desde un sesgo empirista, desde disciplinas de comportamiento aplicado, en orden a lograr como resultado la mayor asistencia a clases (tasas de retención) y el logro de objetivos de aprendizaje.

${ }^{5}$ Department for Education (2017), Effective practice in the delivery and teaching of English and Mathematics to 16-18 year olds. Retrieved from: https://www.gov.uk/government/uploads/system/ uploads/attachment_data/file/662470/English_and_Mathematics_to_16-18_year_olds.pdf

${ }^{6}$ Department for Education -DfE- (2018), The behavioural insights team for adult skills and knowledge, Department for Education. (2018), Retention and success in Maths and English. A practitioner Guide to Applying Behavioural Insights.Available at: https://www.gov.uk/government/publications/ improving-engagement-and-attainment-in-maths-and-english-courses

${ }^{7}$ Bigs, 


\section{Sistematización de experiencia Aprendizajes con anclaje En la convivencia ciudadana Juan Diego Castrillón Cordovez}

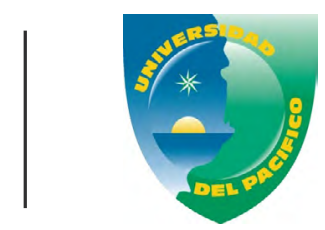

Como alternativa a estos enfoques puede tenerse en cuenta que el aprendizaje puede ser a modo de respuesta a problemas vistos desde el estudiante y que igualmente responda a principios y a hechos empíricos. Un ejemplo de esta alternativa donde se reconocen principios y se orienta desde la voluntad y la razón a resultados empíricos es el ejercicio que a nivel global se ha realizado en el marco de las Naciones Unidas responden a los Objetivos del Desarrollo Sostenible (2015-2030). En la tradición de los pueblos originarios de América estos objetivos pueden ubicarse en la orientación al Buen Vivir que requiere considerar un contexto complementario, pues buena parte de los problemas de los estudiantes que pueden servir de contexto para motivar el aprendizaje responden al entorno local y global.

Más allá del uso de temas y tópicos desde una lectura global como la de Naciones Unidas, como lo advierte la UNICEF Canadá al proponer una pedagogía democrática (Unicef Canadá, 2011:22) ${ }^{8}$ el reto fundamental es realizar prácticas de aprendizaje en escenarios que favorezcan el diálogo respetuoso basado en la lectura reflexiva de marcos teóricos y conceptuales que valoren diferentes perspectivas de pensamiento mientras estén articuladas al reconocimiento y respeto de la dignidad humana. Como reseña el informe de la UNICEF Canadá

En los párrafos siguientes se enuncian algunos principios de aprendizaje que fueron reformulados y se apoyan en una investigación de psicología conductual orientada a valorar diferentes modos de apoyar el aprendizaje en la enseñanza de habilidades en inglés y matemáticas en Inglaterra (DfE,2018) ${ }^{9}$. A cada principio corresponde un ejemplo de aplicación.

Algunas de las prácticas ordenadas al aprendizaje que tienen en cuenta el contexto tanto como dentro como fuera del salón de clases pueden incluir juego de roles, puestas en escena de teatro o danza, aprendizaje basado en problemas, actividades de aprendizaje conversatorios centrados en la participación, debates, exposiciones grupales con responsabilidades evaluables individualmente, simulaciones de pruebas estándar orientadas a evaluar capacidades o competencias, escritos académicos que recojan diversas perspectivas, encuestas de percepción.

Diversos enfoques pedagógicos son posibles a partir de la articulación de diversas propuestas surgidas de las ciencias del comportamiento, sin excluir teorías per se por posiciones ideológicas que renuncien a considerar el aprendizaje centrado en el estudiantes y en el reconocimiento y respeto a la dignidad humana.

La aplicación de prácticas pedagógicas desde principios y resultados puede adaptarse a diversas circunstancias siendo preferible propiciar el aprendizaje desde contextos cercanos a la experiencia y conocimientos previos del estudiante.

Pueden tratarse prácticas constructivistas como apertura a la innovación del conocimiento, pero también las surgidas de la teoría conductista, la cual tiene la limitación de que parten de un sujeto racional que opera en orden a maximizar su bienestar a través regulaciones racionales sociales, con la falla de olvidarse de la complejidad de las historias personales que inciden en los desempeños de los sujetos que participan en ellas. No obstante, los resultados de algunos principios planteados desde una teoría conductista pueden ser efectivos en ambientes muy particulares desde intervenciones objetivas controladas, sin que puedan garantizarse su eficacia en todos los casos.

\footnotetext{
${ }^{8}$ UNICEF Canada, 2011, Educating For Global Citizenship: A Practical Guide for Schools in Atlantic Canada. Consultado en septiembre 23, 2019. Ver también Covell, K., and Brian Howe. Empowering Children: Children's rights education as a pathway to citizenship, Toronto: University of Toronto Press Incorporated, (2005).

${ }^{9}$ Department for Education -DfE- (2018), The behavioural insights team for adult skills and knowledge, Department for Education. (2018), Retention and success in Maths and English. A practitioner Guide to Applying Behavioural Insights.Available at: https://www.gov.uk/government/publications/ improving-engagement-and-attainment-in-maths-and-english-courses
} 
El estudiante tiene particularidades que le diferencian de otros actores y procesos de modo que el producto de aprendizaje pueda lograr una mejor cualificación de sus competencias. Considerando que el reto es que estudiante logre evidenciar sus capacidades o competencias, se pueden tener en cuenta dos dimensiones del aprendizaje significativo según la propuesta de David Ausubel y Joseph Novak: 1. El modo en que se adquiere el conocimiento (por recepción o descubrimiento). 2. Por la incorporación del conocimiento en la estructura cognitiva del estudiante (que puede ser por repetición o significativo).

Adicionalmente puede considerarse el método S.O.L.O. Aplicación del modelo teórico 3P de aprendizaje Biggs, 1987a, 1993a), en el cual se postula que las variables del pronóstico (previas) y del proceso, inciden en las de producto. En consecuencia la ruta metodológica debe orientarse a que puedan detectarse distintos niveles cognitivos de aprendizaje con base en tópicos, contextos y marcos teóricos conceptuales requeridos previamente al estudiante. Los que aquí se muestran son una simplificación de la propuesta de Biggs:

Si la ruta considera niveles de aprendizaje para ser evaluados, significa que las actividades deben orientarse a lograr objetivos específicos por tales niveles. A esta consideración suele llamarse alineación de objetivos del aprendizaje con la evaluación del aprendizaje.

Nivel uniestructural: En este nivel, el objetivo es que estudiante identifique actores, autores agentes sociales, textos, en una situación o fenómeno específico. Establece el significado de un elemento particular, establece datos numéricos, fechas, nombres de lugares. Corresponde al aprendizaje de significados independientes, locales, explícitos o de diccionario que constituyen la base o la entrada a los niveles implícitos del texto.

Nivel multiestructural: En este nivel, el objetivo es que estudiante distinga similitudes y diferencias entre varios actores, autores, agentes sociales, textos, en una situación o fenómeno específico. Entre los elementos varios establece inferencias básicas. Como puede hacer comparaciones y clasificaciones, puede además parafrasear, enlistar, identificar elementos que tienen la función de caracterizar las cosas o las personas.

Nivel relacional: En este nivel el objetivo es establecer conexiones entre antecedentes, causas y/o consecuencias, establecer conjeturas, distinguirlas de los fenómenos reales, encontrar intencionalidades en los actores en distintos fenómenos y distingos marcos contextuales para interpretar.

Nivel abstracto extendido: En este nivel, el objetivo es evidenciar la comprensión desde argumentos que sustenten razonablemente las conclusiones, establecer validez de diversas interpretaciones, y aproximaciones inter disciplinares y culturales, de modo que pueda, además de hacer comparaciones y clasificaciones, concluir, formular propuestas, distinguir intencionalidades, diferenciar antecedentes y causas.

Aunque esta aproximación es válida en la práctica se ha visto insuficiente al reducir el aprendizaje a un plano cognitivo, que no deben entenderse como procesos separados sino interrelacionados e integrados en el proceso de aprendizaje de desarrollo humano

En un enfoque de pedagogía ciudadana, es posible diferenciar también otros modos de aprendizaje que son significativos para la convivencia:

- Además del desarrollo cognitivo, entendido como el conocimiento y el logro de habilidades de pensamiento necesarias como describir, analizar, interpretar, sintetizar, proponer, comprender fenómenos y sus complejidades, se requieren los siguientes enfoques de desarrollo del aprendizaje, diferenciados en la tradición de la educación para la ciudadanía global, según modelos de la Unesco. 


\section{Sistematización de experiencia Aprendizajes con anclaje En la convivencia ciudadana Juan Diego Castrillón Cordovez}

- Desarrollo Socioemocional: valores, actitudes y habilidades sociales que permiten que los alumnos se desarrollen afectiva, psicosocial y física y para permitirles vivir juntos con otros Respetuosa y pacíficamente.

- Desarrollo Conductual, práctico, de desempeño, de aplicación práctica y compromiso.

\section{El proceso con alineación de principios y resultados}

Finalmente, los aprendizajes logrados van más allá del logro de más habilidades, capacidades o talentos de acuerdo de una prueba estándar de aprendizaje, sino que se trata de la formación o apropiación de un modo progresivo de ser para relacionarse con otros y que habilita para un mejor desempeño humano, ciudadano y laboral.

Si tuviera que mostrarse la posibilidad de la experiencia de modo esquemático habría que enumerar el proceso así (UNESCO, 2015) ${ }^{10}$.

3.1. La práctica está centrada en el aprendizaje que promueva competencias (capacidades) prácticas y actitudinales que involucren el sentido de pertenencia y variables socioemocionales, además de capacidades intelectuales que impliquen el análisis, la lectura crítica y la comprensión referida a distintos ámbitos. Este principio implica enfocarse en el avance progresivo de las capacidades de cada uno de los actores de la comunidad educativa, con métodos de formación dialógicos y colaborativos que incluya en la docencia la dimensión personal de los estudiantes, la autonomía en la gestión del aprendizaje y el desarrollo de actitudes críticas y reflexivas.

Implica además promover procesos de reflexión en la acción con fundamentos disciplinares y valorativos (éticos y legales) a partir de advertir la problematización de la realidad vivida. Un ejemplo de aplicación es construir árboles de problemas socioeconómicos en cuanto planteamiento de situaciones por resolver que tienen unas raíces principales y otras secundarias.

Adicionalmente se pueden fomentar interacciones positivas donde se precisen los valores personales y las actividades a realizar en el corto plazo para el mejoramiento del aprendizaje que responda a las motivaciones del estudiante. Un ejemplo de aplicación es el diseño de un plan de desarrollo personal donde el estudiante identifique nombres de proyectos de corto, mediano y largo plazo, relacionados con el ámbito académico, laboral, familiar, recreativo y de servicio cívico. Los nombres de proyectos suelen ser presentados de modo tan genérico e irrealizable que no diferencian tiempo ni lugar de realización, así que el papel del docente es de guía para orientar a los estudiantes a formular proyectos medibles, realizables, financiables, evaluables.

3.2. Se requiere tener en cuenta dominios o campos de aprendizaje y marcos conceptuales. En este ejercicio se plantea la necesidad de considerar conceptos previos del estudiante y valores prácticos de desempeño laboral y valores actitudinales de convivencia que corresponden al marco conceptual del módulo o del curso del estudiante.

Implica favorecer el diálogo interpersonal e interdisciplinar desde la valoración del conocimiento diferenciado como un resultante provisional e incierto que se abre a nuevas formas de comprensión. Un ejemplo de aplicación es presentar en forma escalable y clara el paso a paso de las acciones de aprendizaje a realizar por el estudiante al comienzo de cada sesión y al cierre

10 UNESCO, 2015, Global Citizenship Education: Topics And Learning Objectives, Open Access Repository (http://www. unesco.org/open-access/terms-use-ccbysa-en, 7, place de Fontenoy, 75352 Paris 07 SP, France 
de cada sesión indicar el paso a paso de acciones de aprendizaje independiente y colaborativo fuera de aula y relacionado con los objetivos modulares o del curso. Metódicamente requiere mantener la conexión entre el objetivo de cada actividad con el objetivo del módulo y con el objetivo del curso en el cual fue concebida de modo coherente.

3.3. La evaluación está alineada con los objetivos del aprendizaje tanto del curso como del módulo en el cual figura la sesión de clases. Al respecto, una de las posibilidades evaluativas que se aplicó fue el modelo pedagógico a partir de una Estructura de Resultados Observados de Aprendizaje (Bigs, 2006) ${ }^{11}$ donde se distinguen 4 niveles de competencias intelectivas: El nivel uniestructural, que identifica datos específicos. El nivel multiestructural que permite realizar inferencias dentro de un mismo marco de observación. El nivel relacional que establece conexiones entre eventos que corresponden a distintos marcos de observación, hipótesis, causas, consecuencias, y el nivel abstracto ampliado, que permite emitir juicios desde una posición sustentada, devela posiciones ideológicas y asume diversos correlatos socioculturales o disciplinares y los pone en diálogo.

Implica tomar en cuenta los dominios o niveles de aprendizaje a los cuales corresponden evidencias, atributos claves y tópicos, de acuerdo con la edad del estudiantado. La orientación de la formación participativa con función social, a partir del reconocimiento de la autonomía individual.

Un ejemplo de aplicación es diferenciar contextos, marcos teórico- conceptuales, escenarios y actores específicos en ámbitos locales, regionales y globales cuanto a la perspectiva del alineamiento constructivo y la taxonomía SOLO (sigla en inglés que significa Estructura de Resultados de Aprendizaje Observados) propuesta por Biggs (2006), la cual permite construir evaluaciones teniendo en cuenta los objetivos de aprendizaje y el logro de distintos niveles de complejidad de aprendizaje de los estudiantes, considerando cuatro niveles: uniestructural, multiestructural, relacional y abstracto ampliado

Adicionalmente pueden considerarse otros principios que fueron identificados en una investigación conductista del Departamento de Educación de Inglaterra, Department for Education,DfE,2018) ${ }^{12}$ reseñada antes y que inicialmente tuvo consideración desde aprendizaje de matemáticas en población de riesgo:

3.4. Renovar la motivación al aprendizaje. Es fundamental insistir y alentar al aprendizaje a través de la recurrencia de mensajes con indicaciones del paso a paso a seguir en el proceso, a partir de los cuales el estudiante logre apoyo para asegurar su asistencia y lograr hábitos de estudio. La mensajería de texto es una forma escalable y rentable de proporcionar este soporte. Este principio implica favorecer el diálogo interpersonal e interdisciplinar desde la valoración del conocimiento diferenciado como un resultante provisional e incierto que se abre a nuevas formas de comprensión. Un ejemplo de aplicación es presentar en forma escalable y clara el paso a paso de las acciones de aprendizaje a realizar por el estudiante al comienzo de cada sesión y al cierre de cada sesión indicar el paso a paso de acciones de aprendizaje independiente y colaborativo fuera de aula y relacionado con los objetivos modulares o del curso. Metódicamente requiere mantener la conexión entre el objetivo de cada actividad con el objetivo del módulo y con el objetivo del curso en el cual fue concebida de modo coherente.

\footnotetext{
${ }_{11}^{11}$ Biggs, John,(2005) Calidad del aprendizaje universitario. P.64 Narcea

12 Department for Education -DfE- (2018), The behavioural insights team for adult skills and knowledge, Department for Education. (2018), Retention and success in Maths and English. A practitioner Guide to Applying Behavioural Insights.Available at: https://www.gov.uk/government/ publications/improving-engagement-and-attainment-in-maths-and-english-courses
} 


\section{Sistematización de experiencia Aprendizajes \\ con anclaje En la convivencia ciudadana \\ Juan Diego Castrillón Cordovez}

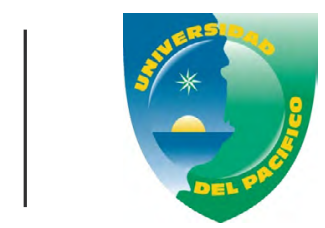

\subsection{Promover redes de apoyo social.}

Muchos alumnos carecen del estímulo continuo necesario para perseverar en sus estudios y suelen desconocer que pueden existir amigos, familiares y conocidos que estarían dispuestos a ayudarles. Estas redes de apoyo pueden movilizarse con gran efecto para mejorar el compromiso con el aprendizaje. La red es tanto de carácter afectivo como académico que responda a las condiciones específicas del estudiante, de modo personalizado. Por eso es importante que él mismo participe en la identificación de los integrantes de la red, que reciba mensajes motivantes y la conmine a mantenerse involucrado y motivado.

Es importante considerar la participación del estudiante en la conformación de su propia red de apoyo orientada por el docente, a quien le corresponde elaborar mensajes motivantes para esta misma red de apoyo y conminarla a mantenerse involucrada y motivada en el aprendizaje de los estudiantes. Este paso complementa la tarea de recordar la agenda del curso al estudiante tanto en clase como en la red mediante mensajes de texto para reforzar el aprendizaje de los procesos y los contenidos principales vistos o pendientes de tratarse.

Este principio implica abrir la experiencia formativa fuera del aula y de los parámetros de las disciplinas, de modo que involucre amigos y parientes de los estudiantes comprometidos con el logro de los objetivos del aprendizaje de cada uno de los estudiantes.

\section{Un ejemplo de aplicación, paso a paso.}

1. Seleccionar con apoyo de cada estudiante la conformación de una red de amigos y familiares que estén en condiciones de motivarlos a la asistencia y al rendimiento en el aprendizaje. 2. Compartir a la red de apoyos los logros por las actividades en las clases (DfE, 2018). 3. Invitar a la red el seguimiento de la agenda formativa, para reforzar el aprendizaje de los procesos y los contenidos principales vistos o pendientes de tratarse. 4. Además de recordar en clase la agenda del curso se requiere el uso de mensajes de texto dirigido particularmente a los estudiantes

El envío de mensajes de texto a los amigos y familiares de los alumnos sugiere que el alumno mejore la asistencia en un 7 por ciento (4.1 puntos porcentuales, del 55.6 al 59.7 por ciento) y el rendimiento en un 27 por ciento (5.9 puntos porcentuales, del 22.2 al 28.1 por ciento). Adicionalmente, los incentivos financieros que requieren la asistencia de un alumno y un compañero nominado mejoraron la asistencia en un 73 por ciento (31.7 puntos porcentuales, de 43.6 a 75.3 por ciento), de acuerdo con el Departamento para la Educación que orientó una investigación desde ciencias conductuales (DfE, 2018)

\subsection{Crear un sentimiento de pertenencia con la realidad académica}

Si las deserciones y renuncias pueden presentarse cuando hay un débil de sentimiento de pertenencia, establecer anclajes con la realidad académica tanto en el aula como fuera del aula es una alternativa. Requiere la combinación de normas sociales y de procesos de auto persuasión de los estudiantes Identificación de prioridades del estudiante. Se ayuda así a identificar motivaciones intrínsecas para el aprendizaje.

Este principio implica Abrir procesos de auto persuasión en los estudiantes para que identifiquen sus prioridades y definan el modo de lograrlas con objetivos medibles de mejoramiento y considerando el impacto para su formación ciudadana y desempeño laboral.

Un ejemplo de aplicación es realizar un diálogo positivo sobre los sentimientos de amenaza o de debilidad en el aprendizaje. Seguidamente de iniciar ejercicios de escritura donde en primera instancia puedan identificar situaciones y experiencias educativas previas que les pueda hacer 
sentirse incómodos en el entorno de aprendizaje. En tercer lugar identificar las normas sociales que favorecen el logro de sus expectativas.

\section{Estudio de caso: Ataque con música a prejuicios y estereotipos}

En tiempos de pandemia ante el riesgo de la monotonía en la virtualidad, el reto asumido en clases en el primer semestre lectivo es sorprender a los estudiantes con artistas de primera talla en clases, como Yella Beezy, -Lot of niggas be talkin' that gang shit-, Piero, -Todos los días-, Héctor Lavoe -Periódico de Ayer- y Chico Buharque - Querido Amigo- al lado de los grupos Radiohead, -Creep-, Supertramp, - The logical song- The Beatles - A day in a life- y el Trío América -El Camino de la Vida-

Luego de la escucha atenta de los videos musicales y de preguntas orientadoras de las lecturas de Pierre Bourdieu y Gianni Vattimo, se programaron en varias sesiones distintas evaluaciones alineadas con el objetivo general y los objetivos específicos del curso de Sociología y Antropología de la Cultura para estudiantes de Comunicación Social en la Universidad Autónoma de Occidente, Cali, que se orienta al reconocimiento de diversos contextos e historias personales, de grupos e instituciones sociales, como medio para la superación de los prejuicios étnicos, de orientación política, religiosa o de género,

Como docente universitario en Cali, Colombia, he sentido la necesidad de promover la escucha activa como competencia básica en el aprendizaje al lado de la lectura y de la escritura. Por eso la invitación de grupos y cantantes es una posibilidad de favorecer la convivencia y quitarle el velo a prejuicios y estereotipos. La música cuando se escucha activamente invita a la interiorización y desde ella a la posición crítica o de lo contrario conduce a mantenerse en la ingenuidad y a someterse a la manipulación.

Para meterle música a las clases hubo de considerarse que la letra de las canciones es de por sí una reflexión, como un espejo que refleja ideales y patologías sociales y puede usarse desde la escucha activa para evidenciar el logro de competencias de aprendizaje, descriptivas, analíticas, relacionales, propositivas y comprensivas. Se usaron canciones populares subtituladas y se contextualizaron para que su análisis particular se hiciera desde contextos y conflictos que los produjeron de modo que fueran más cercanos a los estudiantes.

Metodológicamente estas experiencias se regularon desde agendas de sesiones dadas en conocer en documentos explicativos dispuestos para los estudiantes en plataforma web y que diferenciaban cada una su objetivo específico, su contexto, qué evidencia de aprendizaje se espera del estudiante, qué apoyo se puede esperar del profesor y cuál es la bibliografía disponible en enlaces en la web y la museografía a utilizar disponible en la web de Youtube.

Estas experiencias fueron aplicación de una tesis doctoral en Humanidades en la Universidad del Valle que hice sobre "La escucha de la música como clave para el cambio anímico" y que se basó en la comprensión del libro V del tratado de Aurelio Agustín, De Música. Se asume que la escucha activa favorece la formación ciudadana para interiorizar un discurso que en principio tiene elementos rítmicos, aritméticos, de armonía.

Hubo orientación del profesor en cuento presentación de la agenda por sesiones como también presentación individual de cada uno de los estudiantes distribuidos por grupos y por salas de chat, con un trabajo específico expuesto en la plenaria del curso.

Posteriormente a estas experiencias participativas se hicieron simulaciones de evaluaciones individuales desde la plataforma virtual que volvieron a insistir sobre la adecuada comprensión conceptual y práctica. 


\section{Sistematización de experiencia Aprendizajes con anclaje En la convivencia ciudadana Juan Diego Castrillón Cordovez}

La competencia básica de aprendizaje desde la escucha requiere más teorización y desarrollos aplicativos. Como docente universitario en Cali, Colombia, he sentido la necesidad de promover la escucha activa como competencia básica en el aprendizaje al lado de la lectura y de la escritura. Por eso la invitación de grupos y cantantes es una posibilidad de favorecer la convivencia y quitarle el velo a prejuicios y estereotipos. La música cuando se escucha activamente invita a la interiorización y desde ella a la posición crítica o de lo contrario conduce a mantenerse en la ingenuidad y a someterse a la manipulación.

\section{Aprendizajes desde contextos: Dominios-campos conceptuales y fenómenos sociales}

El aprendizaje requiere anclarse en realidades contemporáneas vistas desde categorías conceptuales que den cuenta de la calidad de su formación y de las competencias desarrolladas por los estudiantes, que tienen que ver con describir, analizar, explicar y comprender tales contextos. La contextualización requiere diferenciar escenarios y tópicos.

Escenarios: Se requiere potenciar los aprendizajes ligados a contextos pertinentes al estudiante, los cuales se definen en escenarios donde además de fenómenos sociales hay pertinencia de aplicar categorías teórico conceptuales para poder

Tópicos. Los tópicos identificados para el aprendizaje requieren precisarse en un contexto local, regional o global que permita la distinción de conflictos y actores cuyo significado puede explicarse y comprenderse de modo más enriquecedor mediante el manejo de un marco teórico conceptual y metodológico.

Para la construcción de contextos de aprendizaje se puede tener en cuenta el enfoque de Van Dijk, en su propuesta de relacionamiento de estructuras sociales y su representación a través contextos, "los cuales sirven para controlar la producción y la recepción del discurso", apropiada a la situación intersubjetiva (Van Dijk, 2001, p. 73). En Van Dijk, "No es la situación social - comunicativa la que influye en las estructuras verbales / discursivas, sino su representación mental en cada participante" (...) y como todo modelo mental, "el modelo del contexto no representa todos los aspectos personales o sociales de la situación comunicativa, sino solamente los aspectos que un momento dado son relevantes para cada participante, sean hablantes u oyentes.)" (Van Dijk, 2001, p. 72)

Una vez el tópico expuesto en su contexto, el reto que se propone al estudiante puede involucrar que evidencie sus capacidades de identificación, diferenciación, comparación, análisis, síntesis, formulación de propuestas y comprensión, con apoyo del marco teórico que corresponde a los contenidos de aprendizaje. Esos contenidos serán más explícitos si se asumen desde los dominios cognitivos prácticos y actitudinales, es decir, si involucran otras dimensiones de la condición humana además de la racional, y que respondan a la interacción humana regida por la voluntad y los afectos.

Dominios mínimos para el aprendizaje en Ciencias Sociales

Para esta enunciación de dominios mínimos se tuvo en cuenta un diagnóstico realizado en el Canadá (UNICEF, 2019) ${ }^{13}$

1. La condición de ciudadanía en la relación de estructuras y sistemas locales y globales.

2. Diferenciación de perspectivas para diferenciar el poder político, económico, social y la responsabilidad frente al uso del poder.

3. Dimensiones de la autoridad, del gobierno, del ámbito del Estado, de las organizaciones civiles.

4. El valor del territorio como referente a sistemas y estructuras locales, regionales y globales.

${ }^{13}$ UNICEF Canada, 2011, Educating For Global Citizenship: A Practical Guide for Schools in Atlantic Canada. 
5. El reconocimiento, la construcción de la identidad personal y colectiva, el sentido de pertenencia. La interacción entre diferentes niveles de identidad para la construcción de una cultura de paz con diferentes grupos sociales.

6. Diferenciación entre similitud y diferencia, buenas relaciones entre diversos individuos y grupos, retos para el respeto a la diferencia, desarrollo y aplicación de valores, actitudes y herramientas para gestionar con diversos grupos y perspectivas.

7. Examinar cómo individuos y grupos han tomado acciones en asuntos en el plano local, regional o global. Desarrollar habilidades para una vinculación ciudadana efectiva

8. Analizar los retos y dilemas asociados con la justicia social y la responsabilidad ética y considerar las implicaciones para la acción individual y colectiva. Toma de posición en orden a la justicia social y a la responsabilidad ética para enfrentar la discriminación y la inequidad.

9. Proponer acciones para llegar a ser un agente de cambio social positivo.

En un enfoque propositivo del aprendizaje se puede invitar a los estudiantes a establecer la conexión de los problemas específicos locales con alternativas de solución global, sobre las cuales existe no solo la enumeración sino el manejo requerible de indicadores para proponer intervenciones de impacto global.

Estos retos se encuentran planteados en la enunciación de los 17 Objetivos de Desarrollo Sostenible (2015-2030):

Poner fin a la pobreza en todas sus formas en todo el mundo.

Poner fin al hambre, lograr la seguridad alimentaria y la mejora de la nutrición y promover la agricultura sostenible.

Garantizar una vida sana y promover el bienestar para todos en todas las edades.

Garantizar una educación inclusiva, equitativa y de calidad y promover oportunidades de aprendizaje durante toda la vida para todos.

Lograr la igualdad entre los géneros y el empoderamiento de todas las mujeres y niñas

Garantizar la disponibilidad de agua y su ordenación sostenible y el saneamiento para todos.

Garantizar el acceso a una energía asequible, segura, sostenible y moderna para todos.

Promover el crecimiento económico sostenido, inclusivo y sostenible, el empleo pleno y productivo y el trabajo decente para todos.

Construir infraestructura resiliente, promover la industrialización inclusiva y sostenible y fomentar la innovación.

Reducir la desigualdad en y entre los países.

Lograr que las ciudades y los asentamientos humanos sean inclusivos, seguros, resilientes y sostenibles.

Garantizar modalidades de consumo y producción sostenibles.

Adoptar medidas urgentes para combatir el cambio climático y sus efectos (tomando nota de los 


\section{Sistematización de experiencia Aprendizajes \\ con anclaje En la convivencia ciudadana \\ Juan Diego Castrillón Cordovez}

acuerdos celebrados en el foro de la Convención Marco de las Naciones Unidas sobre el Cambio Climático).

Conservar y utilizar en forma sostenible los océanos, los mares y los recursos marinos para el desarrollo sostenible.

Proteger, restablecer y promover el uso sostenible de los ecosistemas terrestres, efectuar una ordenación sostenible de los bosques, luchar contra la desertificación, detener y revertir la degradación de las tierras y poner freno a la pérdida de la diversidad biológica.

Promover sociedades pacíficas e inclusivas para el desarrollo sostenible, facilitar el acceso a la justicia para todos y crear instituciones eficaces, responsables e inclusivas a todos los niveles.

Fortalecer los medios de ejecución y revitalizar la alianza mundial para el desarrollo sostenible.

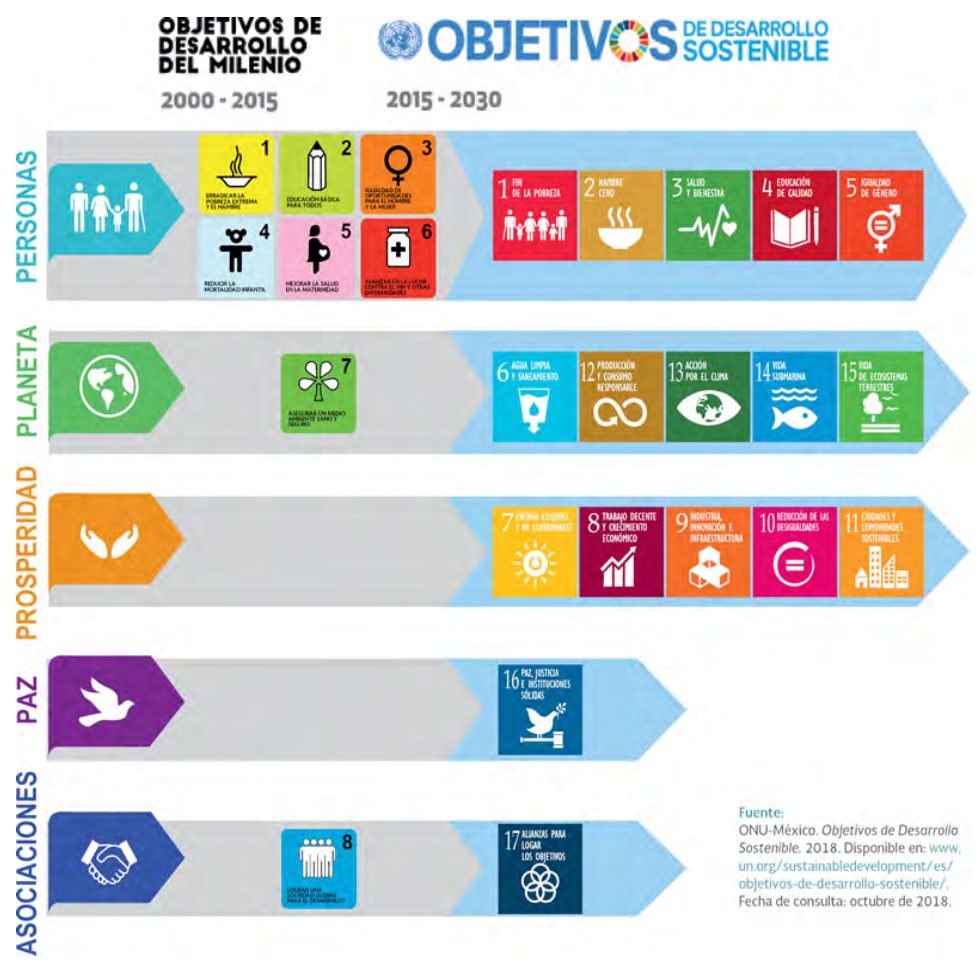

\section{Conclusión}

En materia de los aprendizajes para la convivencia ciudadana en tiempos de conflicto, la selección de la ruta metodológica requiere considerar el punto de partida en el cual se dispone de una previa caracterización del estudiante como actor principal del proceso en función del aprendizaje a través de una alineación de los objetivos y la evaluación. Consideraciones finales:

Establecer un espacio abierto y democrático en clase, determinando los principales intereses, habilidades y expectativas de los estudiantes, invitando al diálogo abierto y al discurso crítico para formular y responder a problemas desde múltiples perspectivas y haciendo conexiones entre eventos y agentes sociales. 
Mantener un contacto regular y alentador con los estudiantes (por ejemplo, a través de mensajes de texto) antes, durante y después de las clases desde redes sociales u otros canales de comunicación.

Se debe da cuenta de la vinculación de la formación con la vida, de modo que se apele a la conversación y a la narración que supere el dogmatismo alrededor de una tesis central y que se abra al reconocimiento de argumentos contrapuestos, o de otras perspectivas que deban ser consideradas.

Se requiere promover la lectura y ofrecer diversidad de apoyos para la experiencia del aprendizaje fuera del aula.

Si bien las comunidades educativas son diversas, es importante considerar estas estrategias porque parten de considerar la permanencia y desempeño en el proceso de aprendizaje dependiendo de la existencia de anclajes del estudiante con la comunidad educativa. Esto implica fomentar compromisos sociales entre compañeros de clase y ayudar a los alumnos a acceder a sus propias redes de apoyo social. 


\section{Referencias bibliográficas}

Berger, P. L. y Luckmann, T. (2003). La construcción social de la realidad. Amorrortu Editores, Argentina.

Bhabha, K. k. (2002). La otra pregunta. El estereotipo, la discriminación y el discurso del colonialismo. Capítulo III. En: El lugar de la Cultura. (pp. 91-110). Editorial Mantial. Buenos Aires.

Biggs, John,(2005) Calidad del aprendizaje universitario. (P.64). Narcea

Delors, J. (1994). "Los cuatro pilares de la educación" En: La Educación encierra un tesoro. pp. (pp. 91 103). México: El Correo de la UNESCO.

Department for Education (2017), Effective practice in the delivery and teaching of English and Mathematics to 16-18 year olds. Retrieved from: https://www.gov.uk/government/uploads/system/ uploads/attachment_data/file/662470/English_and_Mathematics_to_16-18_year_olds.pdf

Department for Education -DfE- (2018), The behavioural insights team for adult skills and knowledge, Department for Education. (2018), Retention and success in Maths and English. A practitioner Guide to Applying Behavioural Insights.Available at: https://www.gov.uk/government/publications/improvingengagement-and-attainment-in-maths-and-english-courses

Fernández Poncela, A. M. (2011). Prejuicios y Estereotipos. Refranes, chistes y acertijos, reproductores y transgresores. En: Revista de Antropología Experimental. N. 11. Texto 22. (pp. 317-328).

Giddens, A. (2006). Interacción Social y vida cotidiana (capítulo 5). En: Sociología. Alianza Editorial (pp. 143-175).

Kottak, Conrad Phillip (2011). Antropología Cultural. Decimocuarta edición. Universidad de Michigan. Mc Graw Hill. (pp. 3-22).

UNESCO, 2015, Global Citizenship Education: Topics And Learning Objectives, Open Access Repository (http://www.unesco.org/open-access/terms-use-ccbysa-en, 7, place de Fontenoy, 75352 Paris 07 SP, France

UNICEF Canada. (2011). Educating For Global Citizenship: A Practical Guide for Schools in Atlantic Canada. Consultado en septiembre 23, 2019. Ver también Covell, K., and Brian Howe. Empowering Children: Children's rights education as a pathway to citizenship, Toronto: University of Toronto Press Incorporated, (2005).

Vargas Llosa, M. (2012). Capítulo 1: La sociedad del espectáculo. (pp. 33-59). Editorial Alfaguara.

Vattimo, Gianni, Zygmunt, B.; Turistas y Vagabundos (1999). En: Baumann, Zygmunt. La globalización: consecuencias humanas. FCE, Buenos Aires. pp 103-133.

Zygmunt, B. El desafío ético de la globalización (2003). En: Revista Colombiana de Sociología N. 21 (pp. 231-235).

Juan Diego Castrillón Cordovez Universidad Autónoma de Occidente https://orcid.org/0000-0002-2499-6215

Doctor en Humanidades, Universidad del Valle. Magister en Ciencias Políticas de la Pontificia Universidad Javeriana de Cali. Licenciado en Filosofía Summa cum Laude Universidad Pontificia Antoniana Roma. Correo: jdcastrillon@uao.edu.co 